\title{
A CHARACTERIZATION OF INTEGRAL CURVES WITH GORENSTEIN HYPERPLANE SECTIONS
}

\author{
KOHJI YANAGAWA \\ (Communicated by Wolmer V. Vasconcelos)
}

\begin{abstract}
We classify a reduced, irreducible and non-degenerate curve $C \subset$ $\mathbb{P}^{r}$ such that its general hyperplane section $C \cap H$ is arithmetically Gorenstein, but $C$ itself is not. These curves are contained in surface scrolls and are closely related to Castelnuovo theory on curves in projective space.
\end{abstract}

\section{INTRODUCTION}

Let $k$ be an algebraically closed field of characteristic 0 . Recently, Huneke and Ulrich [6] proved the following theorem which generalizes Strano's result ([8]) on curves in $\mathbb{P}_{k}^{3}$.

Theorem A ([6, Theorem 3.20]). Let $C \subset \mathbb{P}_{k}^{r}, r \geq 3$, be a reduced, connected and non-degenerate curve which is not contained in a quadric hypersurface. If a general hyperplane section of $C$ is arithmetically Gorenstein, then $C$ itself is arithmetically Gorenstein.

In this paper, we will refine the result above under the additional assumption that $C$ is irreducible. The main results of this paper are the following.

Theorem 0.1. Let $C \subset \mathbb{P}^{r}$ be a reduced, irreducible, non-degenerate curve. Suppose that a general hyperplane section $\Gamma:=C \cap H$ is arithmetically Gorenstein, but $C$ itself is not. Then $\Gamma \subset H \simeq \mathbb{P}^{r-1}$ is contained in a rational normal curve, and $\operatorname{deg} C \equiv 2(\bmod r-1)$.

Theorem 0.2. For a given integer $d \geq r+1$ such that $d \equiv 2(\bmod r-1)$, there is a smooth, irreducible curve $C \subset \mathbb{P}^{r}$ with $\operatorname{deg} C=d$ which is not arithmetically Gorenstein, but its general hyperplane section is arithmetically Gorenstein.

To prove Theorem 0.1, we use M. Green's "Strong Castelnuovo Lemma" (cf. [3]).

The curves constructed in Theorem 0.2 are closely related to Castelnuovo theory on curves in projective space. For example, let $C \subset \mathbb{P}^{r}$ be a nearly Castelnuovo curve (see [1] or [4] for the definition) with $\operatorname{deg} C \equiv 2(\bmod r-1)$. Then $C$ is smooth, non-degenerate and not arithmetically Cohen-Macaulay, but its general hyperplane section is arithmetically Gorenstein.

After proving these, we prove the hypersurface version of the results above.

Received by the editors July 19, 1994 and, in revised form, October 24, 1994.

1991 Mathematics Subject Classification. Primary 13C40, 13H10, 14H45, $14 \mathrm{H} 50$.

Key words and phrases. Gorenstein ring, hyperplane section, Castelnuovo curve, Uniform Position Lemma, surface scroll. 
Theorem 0.3. Let $C \subset \mathbb{P}^{r}, r \geq 3$, be a reduced, irreducible and non-degenerate curve. If its general degree $d(\geq 2)$ hypersurface section $Z$ is arithmetically Gorenstein, then $C$ itself is arithmetically Gorenstein.

If $C$ is not integral, there is a counterexample of Theorem 0.3. See Migliore [7].

\section{Main Results}

Throughout this paper, $k$ is an algebraically closed field of characteristic 0 .

Let $C \subset \mathbb{P}_{k}^{r}$ be an integral and non-degenerate curve, and $\Gamma:=C \cap H$ a general hyperplane section. Denote the homogeneous coordinate ring of $\mathbb{P}^{r}$ (resp. $C$ ) by $S:=k\left[x_{0}, x_{1}, \ldots, x_{r}\right]$ (resp. $\left.A:=S / I_{C}\right)$. Set $m:=\left(x_{0}, x_{1}, \ldots, x_{r}\right)$. We let $x$ be the linear form which defines $H$. Set $S^{\prime}:=S / x S, A^{\prime}:=A / x A$ and $R:=A^{\prime} / H_{m}^{0}\left(A^{\prime}\right) . S^{\prime}$ (resp. $R$ ) represents the homogeneous coordinate ring of $H \simeq \mathbb{P}^{r-1}$ (resp. $\Gamma \subset H$ ).

We denote the Hilbert function of $C$ (resp. $\Gamma$ ) by $H_{C}$ (resp. $H_{\Gamma}$ ). In other words, $H_{C}(n):=\operatorname{dim}_{k} A_{n}$ and $H_{\Gamma}(n):=\operatorname{dim}_{k} R_{n}$ for all $n \in \mathbb{Z}$.

Note that $R$ is a 1 -dimensional Cohen-Macaulay ring, and the Hilbert series of $R$ is given by

$$
F(R, \lambda)=\sum_{n \geq 0} H_{\Gamma}(n) \lambda^{n}=\left(h_{0}+h_{1} \lambda+\cdots+h_{s} \lambda^{s}\right) /(1-\lambda),
$$

where $h_{0}, h_{1}, \cdots, h_{s}$ are certain positive integers. We call the vector $\left(h_{0}, h_{1}, \cdots, h_{s}\right)$ the $h$-vector of $R$ (or $\Gamma$ ). It is well known that $h_{0}=1$ and $\operatorname{deg} C=\operatorname{deg} \Gamma=\sum_{i=0}^{s} h_{i}$.

Lemma 1.1. Let the notation be as above. For a general hyperplane $H$, we have that

(a) $h_{i} \geq h_{1}=r-1$ for all $2 \leq i \leq s-1$.

(b) If $\Gamma \subset H=\mathbb{P}^{r-1}$ is contained in a rational normal curve, then $h_{1}=h_{2}=$ $\cdots=h_{s-1}$ and $h_{s} \leq h_{1}$.

(c) $\Gamma$ is arithmetically Gorenstein if and only if $h_{i}=h_{s-i}$ for each $i$.

Proof. The assertion follows from "Uniform Position Lemma" (cf. [1]) and the Caylay-Bacharach characterization of arithmetically Gorenstein zero-dimensional schemes (cf. [2]).

Suppose that $R$ is Gorenstein. In this case, $A$ is Gorenstein if and only if it is Cohen-Macaulay. Consider the minimal free resolution of $R$ over $S^{\prime}$ :

$$
0 \rightarrow S^{\prime}\left(-n_{r-1}\right) \rightarrow \bigoplus_{i=1}^{b_{r-2}} S^{\prime}\left(-n_{r-2, i}\right) \rightarrow \cdots \rightarrow \bigoplus_{i=1}^{b_{1}} S^{\prime}\left(-n_{1, i}\right) \rightarrow S^{\prime} \rightarrow R \rightarrow 0
$$

We need the following result due to Huneke and Ulrich.

Lemma 1.2 ([6, Corollary 3.24.]). If $C$ is not arithmetically Cohen-Macaulay, we have

$$
n_{r-1}=\min \left\{i \mid\left[H_{m}^{0}\left(A^{\prime}\right)\right]_{i} \neq 0\right\}+r-1=\max \left\{n_{1, i}\right\}+r-1 .
$$

Now we can prove the following theorem. 
Theorem 1.3. Let $C \subset \mathbb{P}^{r}, r \geq 3$, be a reduced, irreducible and non-degenerate curve. Suppose that $\Gamma=C \cap H$ is arithmetically Gorenstein for a generic hyperplane $H$, but $C$ itself is not arithmetically Gorenstein (equivalently, Cohen-Macaulay). Then,

(a) $\Gamma \subset H \simeq \mathbb{P}^{r-1}$ is contained in a rational normal curve.

(b) $\operatorname{deg} C \equiv 2(\bmod r-1)$.

(c) If $\operatorname{deg} C \geq 2 r$, the intersection of the quadrics containing $C$ is a surface scroll.

Proof. (a) From Lemma 1.2 and the "duality" of the free resolution of $R$, it is easy to see that $\min \left\{n_{r-2, i}\right\}=r-1$. Since we may assume that $\Gamma \subset H$ is in linearly general position, $\Gamma$ is contained in a rational normal curve by M. Green's "Strong Castelnuovo's Lemma" (Corollary 3.c.6. of [3]).

(b) Since $\Gamma \subset \mathbb{P}^{r-1}$ is arithmetically Gorenstein and contained in a rational normal curve, the $h$-vector of $\Gamma$ is given by $(1, r-1, r-1, \ldots, r-1,1)$. Hence, we have $\operatorname{deg} C=\operatorname{deg} \Gamma=\sum_{i=0}^{s} h_{i} \equiv 2(\bmod r-1)$.

(c) By (a), $\Gamma$ is contained in a rational normal curve $X_{\Gamma}$. Since $n_{r-1} \geq r+2$ (note that $\operatorname{deg} \Gamma \geq 2 r$ and $r \geq 3$ ), we have $\min \left\{i \mid\left[H_{m}^{0}\left(A^{\prime}\right)\right]_{i} \neq 0\right\} \geq 3$ and $A_{2}^{\prime}=R_{2}$ by Lemma 1.2. Hence the intersection of the quadrics containing $C$ meets $H$ exactly in $X_{\Gamma}$ (note that the defining ideal of a rational normal curve is generated by quadrics). Thus the intersection of the quadrics containing $C$ is a surface $X$ whose general hyperplane section is a rational normal curve, in particular $\operatorname{deg} X=r-1$. So $X$ is a Veronese surface of $\mathbb{P}^{5}$ or a surface scroll (cf. [1]). But easy calculation shows that a non-degenerate curve contained in a Veronese surface of $\mathbb{P}^{5}$ is always projectively normal. So $X$ is a surface scroll.

Corollary 1.4. Let $C \subset \mathbb{P}^{r}$ be a reduced, irreducible and non-degenerate curve with $\operatorname{dim}_{k}\left(I_{C}\right)_{2}<\left(\begin{array}{c}r-1 \\ 2\end{array}\right)$. If a general hyperplane section of $C$ is arithmetically Gorenstein, then $C$ itself is arithmetically Gorenstein.

Proof. If $\operatorname{deg} C \geq 2 r$ (equivalently $\operatorname{deg} C \neq r+1$ ), the assertion follows from Theorem 1.3.(c), since a surface scroll in $\mathbb{P}^{r}$ is defined by $\left(\begin{array}{c}r-1 \\ 2\end{array}\right)$ quadrics. So we may suppose that $\operatorname{deg} C=r+1$. Then we have $\operatorname{dim}_{k}\left(I_{\Gamma}\right)_{2}=\left(\begin{array}{c}r+1 \\ 2\end{array}\right)-H_{\Gamma}(2)=\left(\begin{array}{c}r+1 \\ 2\end{array}\right)-(r+1) \geq$ $\left(\begin{array}{c}r-1 \\ 2\end{array}\right)+1$. By the same argument as in the proof of [6, Theorem 3.20], we see that $\operatorname{dim}_{k} H_{m}^{0}\left(A^{\prime}\right)_{2} \leq 1$ in this case. Hence we have $\operatorname{dim}_{k}\left(I_{C}\right)_{2} \geq \operatorname{dim}_{k}\left(I_{\Gamma}\right)_{2}-1$. It contradicts the assumption $\operatorname{dim}_{k}\left(I_{C}\right)_{2}<\left(\begin{array}{c}r-1 \\ 2\end{array}\right)$.

Next, we describe a curve with Gorenstein hyperplane sections as a divisor of a smooth scroll. For each integer $e \geq 0$, we denote the rational ruled surface $\mathbb{P}\left(\mathcal{O}_{\mathbb{P}^{1}} \bigoplus \mathcal{O}_{\mathbb{P}^{1}}(-e)\right)$ by $X_{e}$. There is an embedding of $X_{e} \hookrightarrow \mathbb{P}^{r}$ as a rational normal scroll if and only if there exists an integer $n>e$ such that $r=2 n-e+1$.

Let $X_{e} \subset \mathbb{P}^{r}$ be a smooth surface scroll. It is well known that the divisor class group of $X_{e}$ is free of rank 2, having as generators the classes $H$ and $L$ of a hyperplane section and a line of the ruling, respectively. The intersection pairing is given by

$$
H \cdot H=r-1, \quad H \cdot L=1, \quad L \cdot L=0,
$$

and the canonical class is

$$
K_{X_{e}} \sim-2 H+(r-3) L
$$


Let $\alpha, \beta$ be two integers. The existence of a reduced and irreducible curve $C \sim$ $\alpha H+\beta L$ on a scroll $X_{e} \subset \mathbb{P}^{r}$ depends on $e$. But, when $e$ is smallest possible (i.e., $e=0$ if $r$ is odd, and $e=1$ if $r$ is even), the condition for these curves to exist is mildest. With this hypothesis, we have the following fact.

Lemma 1.5. Let $\alpha, \beta$ be two integers with $\alpha>0$. Then the following are equivalent.

(a) There exists a smooth, irreducible curve $C \sim \alpha H+\beta L$ on $X_{e}$.

(b) There exists a reduced, irreducible curve $C \sim \alpha H+\beta L$ on $X_{e}$.

(c) $\alpha\left[\frac{r-1}{2}\right]+\beta \geq 0$.

Proof. Follows from [5, V, Corollary 2.18 and 19]. See also [4].

Let $C \subset \mathbb{P}^{r}$ be a reduced and irreducible curve contained in a smooth scroll $X$ such that $C \sim \alpha H+\beta L$. Then we have that

$$
\operatorname{deg} C=\alpha(r-1)+\beta
$$

and

$$
g=\frac{\alpha(\alpha-1)}{2}(r-1)+(\alpha-1)(\beta-1),
$$

where $g$ is the (arithmetic) genus of $C$. It is easy to see that $C$ is non-degenerate if and only if $\operatorname{deg} C=\alpha(r-1)+\beta \geq r$.

Lemma 1.6. Let $C \subset \mathbb{P}^{r}$ be as above. Then $C$ is arithmetically Cohen-Macaulay if and only if $-(r-2) \leq \beta \leq 1$.

Proof. $C$ is arithmetically Cohen-Macaulay if and only if the genus of $C$ is equal to $\pi(d, r)$ (see [1] or [4] for the definition). Hence the assertion follows from easy calculation. See also [1, III Exercise I -4].

If $C$ satisfies the conditions of the previous lemma, $C$ is called a Castelnuovo curve. A Castelnuovo curve $C \subset \mathbb{P}^{r}$ with $\operatorname{deg} C=d$ has the largest genus among integral and non-degenerate curves in $\mathbb{P}^{r}$ with degree $d$.

Let $d \geq r+1$ be an integer such that $d \equiv 2(\bmod r-1)$. From Lemma 1.5, we can take a smooth, irreducible and non-degenerate curve

$$
C \sim n H+2 L, \quad \text { where } \quad n=\frac{d-2}{r-1}
$$

on some surface scroll. Easy calculation shows that the arithmetic genus of $C$ is equal to $\pi(d, r)-1$, and hence $C$ is a typical example of a "nearly Castelnuovo curve" (see [1] or [4] for detail). From Lemma 1.6, $C$ is not arithmetically CohenMacaulay. On the other hand, since $C$ is contained in a surface scroll, a general hyperplane section $\Gamma=C \cap H$ is contained in a rational normal curve. The $h$-vector of $\Gamma$ is given by $(1, r-1, r-1, \cdots, r-1,1)$ by Lemma 1.1.(b). So $\Gamma$ is arithmetically Gorenstein by Lemma 1.1.(c).

Now, we obtain the following.

Theorem 1.7. For a given integer $d \geq r+1$ such that $d \equiv 2(\bmod r-1)$, there is a smooth, irreducible curve $C \subset \mathbb{P}^{r}$ with $\operatorname{deg} C=d$ which is not arithmetically Gorenstein, but its general hyperplane section is arithmetically Gorenstein.

By Lemmas 1.5 and 1.6, we can determine the set of pairs $(\alpha, \beta)$ such that the curve $C \sim \alpha H+\beta L$ satisfies the assumption of Theorem 1.7. 
Example 1.8 ([6, Example 3.26]). Let $B=k\left[x^{8}, x^{7} y, x^{4} y^{4}, x^{3} y^{5}, y^{8}\right]$ and $C:=$ $\operatorname{Proj} B \subset \mathbb{P}^{4}$. The generic hyperplane section $C \cap H$ is arithmetically Gorenstein, but $C$ is not arithmetically Cohen-Macaulay. Note that $\operatorname{deg} C=8 \equiv 2(\bmod 3)$ and $g=3$. There exists a smooth surface scroll $X \subset \mathbb{P}^{4}$ which contains $C$ as $C \sim 4 H-4 L$.

\section{HyPERSURFACE CASES}

In this section, we study a hypersurface version of the results in the previous section. As in Section 1, let $C \subset \mathbb{P}^{r}$ be a reduced, irreducible, non-degenerate curve, and $A$ the projective coordinate ring of $C$.

Let $f \in S_{d}, d \geq 2$, be a general degree $d$ element which defines a hypersurface $F$. Set $A^{\prime}:=A / f A$ and $B:=A^{\prime} / H_{m}^{0}\left(A^{\prime}\right)$. Then $B$ is the projective coordinate ring of hypersurface section $Z:=C \cap F$ in $\mathbb{P}^{r}$.

Remark 2.1. The general degree $d(\geq 2)$ hypersurface section of $C$ is in linearly general position in $\mathbb{P}^{r}$. The proof of the "Uniform Position Lemma" given in [1] is also applicable in the hypersurface case.

We need a result of J. C. Migliore which is a hypersurface version of Lemma 1.2.

Suppose that $Z$ is arithmetically Gorenstein, and consider the minimal free resolution of $B$ over $S$ :

$$
0 \rightarrow S\left(-n_{r}\right) \rightarrow \bigoplus_{i=1}^{b_{r-1}} S\left(-n_{r-1, i}\right) \rightarrow \cdots \rightarrow \bigoplus_{i=1}^{b_{1}} S\left(-n_{1, i}\right) \rightarrow S \rightarrow B \rightarrow 0 .
$$

Lemma 2.2. Let the notation be as above. Suppose that $C$ is not arithmetically Cohen-Macaulay and $Z$ is arithmetically Gorenstein. Set

$$
b:=\min \left\{n \mid H_{m}^{0}\left(A^{\prime}\right)_{n} \neq 0\right\}=\min \left\{n \mid A_{n}^{\prime} \neq B_{n}\right\} .
$$

Then we have

$$
n_{r}=b+r=\max \left\{n_{1, i}\right\}+r .
$$

Proof. The assertion follows from [7, Proposition 2.2]. See also the argument following (3.2) of [7].

Theorem 2.3. Let $C \subset \mathbb{P}^{r}, r \geq 3$, be a reduced, irreducible and non-degenerate curve. If its general degree $d(\geq 2)$ hypersurface section $Z$ is arithmetically Gorenstein, then $C$ itself is arithmetically Gorenstein.

Proof. Assume the contrary (i.e., $C$ is not arithmetically Cohen-Macaulay). Note that $\operatorname{deg} C \geq r+1$ (if $\operatorname{deg} C \leq r$, then $C$ is a rational normal curve and projectively normal).

From Lemma 2.2 and an argument similar to our proof of Theorem 1.3 (a), we can see that $Z$ is contained in a rational normal curve of $\mathbb{P}^{r}$. Hence we have that $H_{Z}(n)=\min \{\operatorname{deg} Z, n r+1\}$ for all $n \in \mathbb{Z}$.

Case 1. $b=\min \left\{n \mid A_{n}^{\prime} \neq B_{n}\right\}>2$ : Since the defining ideal of a rational normal curve is generated by quadrics, we have $\left(I_{Z}\right)_{2} \neq\left(I_{C}\right)_{2}$ (i.e., $A_{2} \neq B_{2}$ ). By the assumption that $b \geq 3$, we have $d=2$. Easy calculation shows that $H_{C}(2)=H_{Z}(2)+1=2 r+2$. 
Let $x \in S_{1}$ be a general linear form which defines a hyperplane $H$, and set $\Gamma:=C \cap H$. Then,

$$
\begin{aligned}
H_{\Gamma}(2) & =H_{C}(2)-H_{C}(1)-\operatorname{dim}_{k}\left[H_{m}^{0}(A / x A)\right]_{2} \\
& =2 r+2-(r+1)-\operatorname{dim}_{k}\left[H_{m}^{0}(A / x A)\right]_{2} \\
& =r+1-\operatorname{dim}_{k}\left[H_{m}^{0}(A / x A)\right]_{2} .
\end{aligned}
$$

On the other hand, $\Gamma \subset H$ is in linearly general position, and hence $H_{\Gamma}(2) \geq$ $\min \{\operatorname{deg} \Gamma, 2 r-1\}$. So we have $\operatorname{deg} C=\operatorname{deg} \Gamma=H_{\Gamma}(2)=r+1$ and $\left[H_{m}^{0}(A / x A)\right]_{2}=$ 0 . Since $\Gamma$ is non-degenerate in $H$, we have $\left[H_{m}^{0}(A / x A)\right]_{i}=0$ for all $i \leq 1$. Furthermore, since the defining ideal of $\Gamma \subset H$ is generated by quadrics (note that $\Gamma$ is arithmetically Gorenstein with $h$-vector $(1, r-1,1))$, we have $\left[H_{m}^{0}(A / x A)\right]_{i}=0$ for all $i \geq 3$. So we have $H_{m}^{0}(A / x A)=0$, and $A$ is Cohen-Macaulay. This is a contradiction.

Case 2. $b=2$ : Since $\max \left\{n_{1, i}\right\}=b=2$ and $Z$ is contained in a rational normal curve, the $h$-vector of $Z$ is $(1, \mathrm{r}, 1)$ and $\operatorname{deg} Z=d \operatorname{deg} C=r+2$. It contradicts the facts that $d \geq 2$ and $\operatorname{deg} C \geq r+1$.

\section{ACKNOWLEDGMENT}

The author is grateful to Professor J. C. Migliore for valuable e-mail discussion, and sending me the preprints including [7].

\section{REFERENCES}

1. E. Arbarello, M. Cornalba, P. Griffiths and J. Harris, Geometry of Algebraic Curves, vol. 1, Springer-Verlag, 1985. MR 86h:14019

2. E. Davis, A. V. Geramita and F. Orecchia, Gorenstein algebras and the Cayley-Bacharach theorem, Proc. Amer. Math. Soc. 93 (1985), 593-597. MR 86k:14034

3. M. Green, Koszul cohomology and the geometry of projective varieties, J. Diff. Geom. 19 (1984), 125-171. MR 85e:14022

4. J. Harris, Curves in Projective Space, University of Montreal Press, 1982. MR 84g:14024

5. R. Hartshorne, Algebraic Geometry, Springer-Verlag, 1977. MR 57:3116

6. C. Huneke and B. Ulrich, General hyperplane sections of algebraic varieties, J. Alg. Geom. 2 (1993), 487-505. MR 94b:14046

7. J. Migliore, Hypersurface sections of curves, Proceeding of the conference on Zero-dimensional Schemes (Ravello, 1992), De Gruyter, 1994, pp. 269-282. CMP 94:17

8. R. Strano, A characterization of complete intersection curves in $\mathbb{P}^{3}$, Proc. Amer. Math. Soc. 104 (1988), 711-715. MR 90b:14062

Department of Mathematics, School of Science, Nagoya University Chikusa-ku, NAGOYA 464 JAPAN

E-mail address: yanagawa@math.nagoya-u.ac.jp 